\title{
Femtosecond Laser Desorption of Thin Polymer Films from a Dielectric Surface
}

\author{
L. Mercadier ${ }^{1, *}$, J. Peng ${ }^{1}$, Y. Sultan ${ }^{2}$, T. Davis ${ }^{3}$, D. M. Rayner ${ }^{1}$ and P. B. Corkum ${ }^{1}$ \\ ${ }^{1}$ Joint University of Ottawa/National Research Council Laboratory for Attosecond Science, 100 Sussex Dr., Ottawa, ON K1 A 0R6, Canada \\ ${ }^{2}$ Emerging Priorities Division, Environment Canada, Gatineau, QC K1A OH3, Canada \\ ${ }^{3}$ Science and Technology, Canadian Space Agency, Gov. Liaison Office, 240 Sparks St., Ottawa, ON K1A 1A1, Canada \\ *laurent.mercadier@nrc-cnrc.gc.ca
}

\begin{abstract}
We desorb polymer films from fused silica with a femtosecond laser and characterize the results by atomic force microscopy. Our study as a function of beam geometry and energy reveals two ways of achieving spatially controlled nanodesorption.
\end{abstract}

\section{Introduction}

The use of femtosecond laser pulses at critical intensity, i.e. slightly above the threshold for nonlinear absorption, allows absorption that can be triggered in a sub-diffraction volume, opening doors to nanoscale machining [1]. Besides machining, the nanoscale desorption of surface layers has potential technical significance. However, the mechanisms involved in the absorption of the laser energy strongly depend on the laser intensity, and a small fluctuation of the latter can lead to large differences in the induced modifications of the material.

We show that the desorption of thin polymer films from fused silica can be controlled by tightly focusing the femtosecond laser beam through the substrate, thus desorbing from the back surface. We show two approaches to achieve this control: i) by focusing at the surface and optimizing the energy and number of pulses applied, and ii) by focusing inside the substrate and making use of the self-controlled deposition of energy by nonlinear absorption in the wide-band-gap material.

\section{Experimental setup and methods}

We performed desorption of a multilayered thin film of polymer with femtosecond laser pulse. The sample was made of a fused silica $25 \times 25 \times 0.22 \mathrm{~mm}$ microscope coverslip, on which we deposited 4 bilayers of Poly(allylamine hydrochloride) / Poly(sodium 4-styrenesulfonate). Prior to the deposition of the film, gold lines of $200 \mu \mathrm{m}$ width and $100 \mathrm{~nm}$ thickness, each separated by $200 \mu \mathrm{m}$ were deposited on the surface of the coverslip. Desorption was achieved in between the lines. The sample was positioned on three motorized translation axes. We used a Ti:Sa laser delivering pulses of $45 \mathrm{fs}$ duration at a wavelength of $800 \mathrm{~nm}$ and a repetition rate of $50 \mathrm{~Hz}$ and we varied its energy by means of a polarizer and a half-wave plate. The energy fluctuations were on the order of $2 \% \mathrm{RMS}$.

Three objective lenses with $0.25,0.40$ and 0.95 numerical aperture (NA), respectively, were used to focus the laser beam onto the back surface of the sample. The position of the focus with respect to the sample surface was varied by moving the sample along the optical axis $z$.

We used the sharp edges of the gold lines for in situ measurements of the beam waist by the so-called knife-edge technique [2], which enabled us to determine both the focus position and the beam waist with a $0.1 \mu \mathrm{m}$ precision.

\section{Results}

\section{Direct desorption}

The desorption experiment consisted in a matrix of irradiation sites separated by $3 \mu \mathrm{m}$ each. In one direction, we varied the laser energy, while in the other direction, we varied the $z$-position of the focus. The observation of the matrix with AFM enabled characterization of the surface with nanometer resolution. When the energy was below the threshold for fused silica damage and above the threshold for desorption, we observed holes with sharp edges and reproducible $8 \mathrm{~nm}$ depth, clearly indicating that only the film was removed by the fs laser pulse. The diameter of the holes was at maximum when the focus was right at the surface, as shown in fig 1.a.

We found a threshold for desorption of $25 \mathrm{~nJ}$ using the NA 0.40 lens, which corresponds to an intensity of $8 \times 10^{12} \mathrm{~W} / \mathrm{cm}^{2}$ according the beam waist measurement. This threshold is by a factor of two lower than the threshold for non linear absorption in fused silica, which we measured by looking at the transmitted energy as a function of input energy of the focalized beam in the bulk, as done in previous work [3]. Even if this threshold is lowered 
because of the constructive interferences of the incident and reflected fields at the dielectric - air interface, we found that the absorption of energy still occurs directly in the film itself.
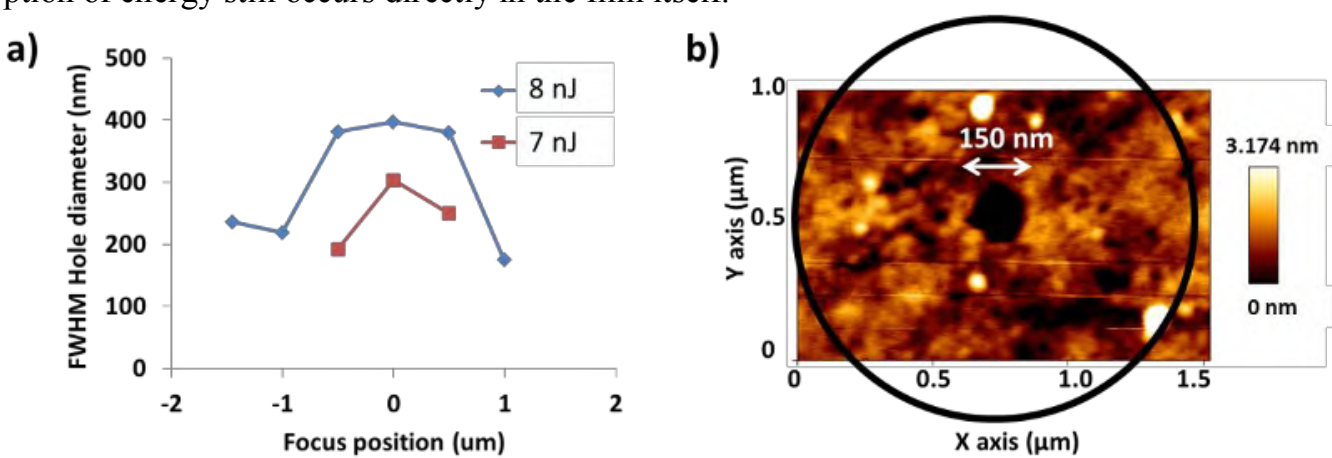

Fig. 1.a) Diameter of the desorbed holes by 10 laser pulses as a function of focus position with respect to the surface for 7 and $8 \mathrm{~nJ}$ pulse energy, with NA 0.95 focusing lens. b) AFM image of the desorbed feature with $7 \mathrm{~nJ}$. The black circle indicates the diameter at $1 / \mathrm{e}^{2}$ of the beam.

The smallest feature observed was $150 \mathrm{~nm}$ in diameter (FWHM), using the NA 0.95 objective (see fig.1.b). However, these small holes were not reproducible using a single laser pulse, mainly due to the instabilities of the laser intensity.

To average out the intensity fluctuations and obtain reproducible desorption features, we increased the number of laser pulses per irradiation site. We found that the diameter increased as a function of the applied number of laser pulses, so that 10 pulses per site was a good tradeoff between small size and reproducibility. We could obtain reproducible $200 \mathrm{~nm}$ diameter desorbed holes, which is by a factor of 6 less than the beam diameter at $1 / \mathrm{e}^{2}$.

\section{Substrate mediated desorption}

The second way of controlling the film desorption is by taking advantage of the nonlinear interaction inside the substrate. It has been shown that filamentation inside dielectrics leads to an elongated damage along the beam propagation axis with small radial dimension [4].

We focused the laser a few Rayleigh ranges back inside the substrate, with pulse energies above the absorption threshold and a power above the critical power for self-focusing. We found that for a specific position of the focus with respect to the surface, it is possible to induce desorption of the film without apparent damage of the substrate surface. In addition, this desorption becomes extremely reproducible even for laser energy variations as large as $30 \%$. For instance, with NA 0.40 , we could observe consistent holes of $500 \mathrm{~nm}$ diameter when varying the energy from 150 to $200 \mathrm{~nJ}$. Although the features observed were bigger, we could achieve a better control and better reproducibility with single pulse irradiation than when focusing right at the surface.

\section{Conclusion}

We performed nanodesorption of polymer thin films from a fused silica substrate by tightly focusing a femtosecond laser on the sample back surface. On one hand, we showed that when working close to the desorption threshold, the substrate plays no role and the film can therefore be considered as free standing. This has potential for strong field interactions with two dimensional structures. On the other hand, we achieved spatial control by inducing filamentation in the substrate prior to desorption, substantially increasing the reproducibility. Both approaches allow desorption on scales below the self-diffraction limit.

\section{References}

[1] A. P. Joglekar et al., Optics at critical intensity: Applications to nanomorphing, PNAS 101 (2004) 5856-5861.

[2] J. J. Chapman et al., An automated submicron beam profiler for characterization of high numerical aperture optic, Rev. Sci. Instr. 79 (2008) 095106-095106-4

[3] D. Grojo et al., Exciton-seeded multiphoton ionization in bulk $\mathrm{SiO}_{2}$, Phys. Rev. B 81 (2010) 212301

[4] A. Couairon et al., Filamentation and damage in fused silica induced by tightly focused femtosecond laser pulses, Phys. Rev. B 71 (2005) 125435 